\title{
El Inventario de Estrategias de futorregulación: traducción al español, características psicométricas preliminares y su relación con variables sociodemográficas en una muestra de estudiantes universitarios
}

\author{
The Self-Regulation Strategy Inventory: Spanish Translation, Preliminary \\ Psychometric Characteristics and its Relation to Sociodemographic Uariables \\ in Sample of University Students
} 0 Inventário de Estratégias de futorregulação: tradução ao espanhol, características
psicométricas preliminares e a sua relação com variáveis sociodemográficas
em uma amostra de estudantes universitários

David Monge-López*, Roger Bonilla**, Wendy Aguilar-Freyan*

*Universidad Hispanoamericana, San José, Costa Rica.

**Universidad de Costa Rica, San José, Costa Rica.

Doi: http://dx.doi.org/10.12804/revistas.urosario.edu.co/apl/a.3729

\section{Resumen}

Esta investigación presenta la traducción al español del Inventario de Estrategias de Autorregulación. El constructo de aprendizaje autorregulado posee importancia como potencial predictor del logro académico. En este trabajo se abordaron estudiantes universitarios y se evaluó el autoinforme de autorregulación al aprendizaje de modo general, con el objetivo de poseer una línea base acerca de la percepción de ellos del proceso en cuestión. El instrumento se aplicó a una muestra de 282 estudiantes. El análisis factorial exploratorio dio como resultado tres dimensiones, las cuales se encuentran también en la investigación antecedente. La medición posee buena fiabilidad, y tanto las dimensiones del instrumento como la medición general de aprendizaje autorregulado correlacionaron con una medida independiente de autorregulación. Con un objetivo exploratorio, se relacionaron la medición de aprendizaje autorregulado con una serie de variables sociodemográficas. Se encontraron asociaciones de diferentes dimensiones del aprendizaje autorregulado con el sexo y el tipo de estructura familiar del estudiante. La calidad del instrumento indica que puede ser utilizado para la investigación sobre el tema.

* David Monge-López, Wendy Aguilar-Freyan, Facultad de Ciencias de la Salud, Carrera de Psicología, Universidad Hispanoamericana, San José, Costa Rica; ${ }^{* *}$ Roger Bonilla, Facultad de Ciencias Económicas, Escuela de Estadística, Universidad de Costa Rica, San José, Costa Rica. Estadístico consultor.

La correspondencia relativa a este artículo debe ser enviada a David Monge-López, Facultad de Psicología, Universidad Hispanoamericana. Sede Barrio Aranjuez. San José, Costa Rica. Teléfono: (506) 22568197. Correos electrónicos: dmonge@uh.ac.cr, davidm179@gmail.com.

Cómo citar este artículo: Monge-López, D., Bonilla, R., \& Aguilar-Freyan, W. (2017). El Inventario de Estrategias de Autorregulación: traducción al español, características psicométricas preliminares y su relación con variables sociodemográficas en una muestra de estudiantes universitarios. Avances en Psicología Latinoamericana, 35(1), 61-78. doi: http://dx.doi.org/10.12804/ revistas.urosario.edu.co/apl/a.3729 
Los resultados obtenidos con respecto al sexo y al tipo de familia deben ser replicados por otros estudios con muestras y métodos similares.

Palabras clave: aprendizaje autorregulado; instrumentos; análisis factorial.

\section{fibstract}

This research presents the translation into Spanish of the Self-Regulation Strategy Inventory. The self-regulated learning construct is important as a potential predictor of academic achievement. In this work, university students assessed their self-regulated learning in a general sense and these assessments were evaluated in order to establish a baseline regarding their own perception of the process. After the test instrument was applied to a sample of 282 students, an exploratory factorial analysis demonstrated three dimensions, all of which were also found in previous research. The measurement is highly reliable; both the dimensions of the instrument and the general measurement of self-regulated learning correlate with an independent measure of self-regulation. As an exploratory exercise, the measurement of self-regulated learning was related to a series of sociodemographic variables. Associations were found between different dimensions of self-regulated learning and students' sex and type of family structure. The quality of the instrument indicates that it can be used for research on this topic. The results with respect to sex and family structure should be replicated in other studies with similar samples and methods.

Key words: Self-regulated learning; instruments; factorial analysis.

\section{Resumo}

Esta pesquisa apresenta a tradução ao espanhol do Inventário de Estratégias de Autorregulação. O construto de aprendizagem autorregulado possui importância como potencial preditor do desempenho académico. Neste trabalho se têm feito uma aproximação a estudantes universitários e se tem avaliado o auto-informe de autorregulação à aprendizagem de modo geral, com o objetivo de possuir uma linha base acerca da autoperceção do processo em questão. $\mathrm{O}$ instrumento se tem aplicado a uma amostra de 282 estudantes. A análise fatorial exploratória tem dado como resultado três dimensões, as mesmas encontradas na pesquisa antecedente. A medição possui boa fiabilidade e as dimensões do instrumento assim como a medição geral de aprendizagem autorregulado têm correlacionado com uma medida independente de autorregulação. Com um objetivo exploratório, se têm relacionado a medição da aprendizagem autorregulado com uma série de variáveis sociodemográficas. Se têm encontrado associações de diferentes dimensões da aprendizagem autorregulada com o sexo e o tipo de estrutura familiar do estudante. A qualidade do instrumento indica que o mesmo pode ser utilizado pela investigação sobre o tema. Os resultados obtidos com respeito ao sexo e o tipo de família devem ser replicados por outros estudos com amostras e métodos similares.

Palavras-chave: aprendizagem autorregulada; instrumentos; análise fatorial.

\section{Introducción}

La noción de aprendizaje autorregulado (AAR) incluye todas aquellas estrategias por medio de las cuales un estudiante es capaz de guiar sus procesos de búsqueda de información y elaboración de conocimiento; implica la habilidad para establecer metas propias, hacer uso de capacidades metacognitivas, sostener la motivación hacia la tarea y obtener realimentación de ésta para modificar el comportamiento según la situación (Boekaerts, Pintrich, \& Zeidner, 2000; Boekaerts \& Corno, 2005).

Según Zimmerman (1990), dentro de las características que poseen los estudiantes que autorregulan su proceso de aprendizaje están incluidas capacidades cognitivas como la tendencia a pensar en alternativas posibles para resolver problemas, el conocimiento de las propias competencias y la habilidad para establecer relaciones estratégicas con el medioambiente. En el plano motivacional, 
los estudiantes con capacidades de autorregulación pueden aceptar las responsabilidades por su propio proceso de aprendizaje y persisten en la tarea hasta conseguir sus objetivos.

En el plano conductual, se trata de estudiantes que buscan información por sí mismos, crean sus propios ambientes para aprender y buscan obtener experiencias positivas de éstos (Kolovelonis, Goudas, \& Dermitzaki, 2011; Labuhn, Zimmerman, \& Hasselhorn, 2010); asimismo buscan consejo y apoyo en caso de ser requerido (Clarebout, Horz, Schnotz, \& Elen, 2010). En síntesis, el AAR es el proceso por el cual un estudiante puede manejar sus propios pensamientos, comportamientos y emociones para maximizar su capacidad de aprendizaje.

ElAAR se ha relacionado con la motivación al logro académico y con el logro académico, con la facilitación del aprendizaje, con la puesta en práctica de hábitos de estudio, el automonitoreo y la autoevaluación del progreso académico (de Bruin, Thiede, Camp, \& Redford, 2011; Harris, Friedlander, Sadler, Frizzelle, \& Graham, 2005; Jarvela \& Jarvenoja, 2011; Kistner et al., 2010; Schunk \& Zimmerman, 2007; Wolters, 2011; Zimmerman, 2008). La relación entre los procesos del AAR, el logro académico y la autoeficacia parecen ser determinantes en el éxito educativo (Cazan, 2012; Bandura, 1997; Pintirch, 2003; Pintrich \& Schunk, 2002; Zuffianò et al., 2013).

Para Zimmerman (2002) la evaluación del AAR resulta importante sobre todo cuando se va progresando en niveles académicos, especialmente al llegar a la secundaria, estudios técnicos o universitarios, ya que en estos niveles aumentan la carga de responsabilidades para el estudiante, el estrés, las múltiples demandas de varios profesores, $\mathrm{y}$ pueden darse problemas en el manejo de la independencia y en la reducción de la motivación. Los estudiantes que presenten dificultades para manejar el tiempo y afrontar situaciones estresantes de la vida académica, y que tengan un pobre despliegue de estrategias de aprendizaje efectivas podrán tener mayor riesgo de presentar problemas académicos (Schunk, 2005).

Boakerts y Corno (2005) hacen un resumen de las diferentes estrategias que se han utilizado para estudiar el AAR hasta mediados de la década pasada, las cuales han permitido predecir fenómenos como los descritos en el párrafo anterior. Los principales tipos de instrumentos utilizados han sido los autoinformes basados en escalas Likert, observaciones de comportamientos, entrevistas, expresión de pensamientos en voz alta, tareas que muestran procesos o eventos mentales autorregulatorios, situaciones manipuladas experimentalmente, grabaciones de estrategias motivacionales en el trabajo y diarios. Por medio de estas estrategias el concepto de AAR genera una diversidad importante de investigaciones.

Algunos de los temas recientemente abordados incluyen la relación entre el AAR y los procesos relacionados con la adquisición de habilidades para el aprendizaje en línea o mediado por computadora (Kuo, Walker, Schroder, \& Belland, 2014; Lehamann, Hähnlein, \& Ifenthaler, 2014; Saks \& Leijen, 2014; Taub, Azevedo, Bouchet, \& Khosravifar, 2014; Won You \& Kang, 2014); el rol de los educadores o el papel de los procesos de enseñanza en la adquisición de habilidades (Endedijk, Brekelmans, Verloop, Sleegers, \& Vermunt; 2014; Peeters et al., 2014); la descripción de variables relacionadas con el AAR en niveles educativos específicos (por ejemplo, primaria, secundaria o educación superior) (Kindekens et al., 2014; Nandagopal \& Anders, 2012; Ozan, Gundogdu, Bay, \& Celkan, 2012; Vandevelde, Van Keer, \& Rosseel, 2013) o en dominios específicos (por ejemplo, la matemática o las ciencias) (Friedrich, Jonkmann, Nagengast, Schmitz, \& Trautwein, 2013; Greene, Hutchinson, Costa, \& Crompton, 2012). También se ha estudiado el AAR y algunos procesos relacionados con éste en contextos educativos no occidentales (Babakhani, 2014; Hadi, Kalantari, \& Ghaslani, 2014). 
En el contexto hispanoamericano el estudio del AAR también ha generado interés en la última década; un resumen de una parte de la investigación al respecto puede revisarse en Rosário et al. (2013). Estos autores han sistematizado la información de diferentes estudios sobre el AAR, específicamente en relación con la naturaleza del AAR, su evaluación, la promoción de habilidades para alcanzarlo, su utilidad en procesos educativos y la implicación de los profesores en la facilitación del AAR. Entre varios aspectos relevantes se señala que se ha dado un incremento paulatino en las publicaciones sobre AAR desde mediados de la década pasada, pero se considera que la investigación en países hispanos está en un momento inicial.

El presente trabajo tuvo como principal fin evaluar las características preliminares de una medición de autoinforme sobre AAR presentada por Cleary (2006); se trata del Inventario de Estrategias de Autorregulación-Autoinforme (SRSI-SR por sus siglas en inglés) y fue originalmente aplicado a estudiantes de niveles avanzados de secundaria. Si bien es cierto, como señalan Rosário et al. (2013), que deberían desarrollarse mediciones del AAR que no solamente recurran al autoinforme, tal tipo de instrumentos se continúan usando mucho en la investigación y en la evaluación de procesos psicológicos.

Cleary (2006) aplicó el SRSI-SR solicitando a los estudiantes que completaran el autoinforme pensando en su desempeño en las ciencias. Los resultados de los análisis factoriales exploratorios mostraron tres dimensiones principales: "búsqueda y aprendizaje de información” (BAI), "manejo del ambiente y el comportamiento" (MAC) y "comportamientos regulatorios maladaptativos" (CRM).

El autor encontró también una alta consistencia (coeficiente alfa $=0,92$ ); una adecuada validez convergente y discriminante; una relación entre género y la dimensión MAC (las mujeres tuvieron puntuaciones significativamente mayores en tal dimensión); un efecto de interacción entre el género y el nivel educativo para los CRM (dicha dimensión decreció del noveno al décimo grado solamente entre las mujeres); relaciones del nivel de logro académico en las tres dimensiones establecidas (una relación negativa sobre la dimensión de CRM y relaciones positivas con las otras dos dimensiones) y relaciones positivas entre variables motivacionales y las dimensiones BAI y MAC. Trabajos posteriores han continuado utilizando el SRSI-SR para evaluar áreas como la matemática (Cleary \& Chen, 2009) o como una medida en estudios de caso pretest-postest (Cleary \& Platten, 2013).

En este estudio se realizó una traducción al español del SRSI-SR y se aplicó el instrumento a una muestra a conveniencia de estudiantes universitarios costarricenses. No obstante, a diferencia del estudio previo antes referido, la aplicación no se circunscribió a un dominio académico específico, sino que se solicitó a los estudiantes que evaluaran sus estrategias de autorregulación de modo general. Esta modificación fue necesaria debido a que el interés del trabajo fue poseer una medición de AAR general que pudiera ser aplicada a distintas áreas de estudio, por lo que la muestra provino de diferentes carreras.

El conocimiento sobre la autoevaluación general que los estudiantes hacen de sus procesos de AAR constituye, desde nuestro punto de vista, un primer paso de capital importancia. Éste debe realizarse antes de estudiar el modo en que se manifiesta el AAR en dominios específicos, debido a que la primera información puede servir de línea base para posteriores estudios sobre áreas concretas, de ahí la gran importancia de trabajos como el presente. Resulta entonces de fundamental relevancia establecer las características del autoinforme general de AAR para que, estudios posteriores, puedan tener un referente previo y comparar los resultados.

Como un objetivo complementario se asoció el AAR con una serie de variables sociodemográficas con un fin básicamente exploratorio. Con excepción de la variable sexo (Cleary, 2006), la caracterización del perfil sociodemográfico no 
suele incluirse en estudios previos sobre el tema, por lo que existe poca información sobre si otras variables de tal tipo se relacionan con el AAR. La importancia de este objetivo complementario radica en que podría permitir la identificación de características asociadas al AAR que previamente no han sido trabajadas, o bien facilitaría descartar tales variables como características no relevantes para el tema en cuestión, al menos con muestras como las abordadas en el presente trabajo.

\section{Método}

El presente fue un estudio transversal por medio del cual se evaluaron algunas características psicométricas del SRSI-SR; en concreto, interesó obtener evidencia tanto sobre la estructura factorial de la medición en cuestión, como sobre la fiabilidad de la medida general de AAR, sus dimensiones y las correlaciones de estas últimas con una medida de autorregulación general que ha funcionado como criterio de validez externa.

Se exploró además si existía relación entre la medida general de AAR o sus dimensiones con algunas variables sociodemográficas, con el objetivo de indagar la existencia de posibles asociaciones no trabajadas previamente en la literatura sobre el tema y que, en caso de existir, podrían sugerir situaciones que coadyuvan o impiden la autoevaluación de AAR.

\section{Participantes}

Se contó con una muestra de 282 estudiantes de diferentes carreras, 47,18\% fueron varones. La edad de los participantes tuvo un rango de 382 meses $(\mathrm{M}=2,7$ años o 285,1 meses, $\mathrm{DT}=62,41$ meses). Con respecto a las carreras, un 10,95\% de los participantes cursaba Psicología, un 49,12\% cursaba Enfermería, un 19,08\% cursaba Informática, un $8,48 \%$ cursaba Administración, un 2,83\% pertenecía a Ingeniería Industrial, un $0,71 \%$ estudiaba Publicidad, un $0,35 \%$ cursaba Turismo, un 2,47\% estudiaba Nutrición, un 3,18\% cursaba Medicina y un 2,83\% estudiaba Arquitectura.

\section{Instrumentos}

\section{Inventario de Estrategias de Autorregulación- Autoinforme (SRSI-SR)}

Como se señaló, este instrumento fue desarrollado por Cleary (2006). Es una escala tipo Likert que consta de 28 ítems. En el estudio original ha presentado una alta consistencia interna $(\alpha=0,92)$. Para esta versión se elaboraron siete opciones de respuesta para cada ítem, las cuales estaban entre estar "Totalmente en desacuerdo" con la afirmación planteada, hasta estar "Totalmente de acuerdo".

\section{Cuestionario de autorregulación general}

Se utilizó una traducción del cuestionario de autorregulación general (ARG) presentado por Kirk y Martin (1998). Se escogió ese instrumento debido a que estaba constituido por un número relativamente pequeño de ítems. El propósito de su inclusión fue la posibilidad de utilizarlo como un criterio externo concurrente para el SRSI-SR. La escala está compuesta por 23 ítems tipo Likert y en el estudio previo presentó una consistencia interna adecuada tanto para mujeres $(\alpha=0,83)$ como para hombres $(\alpha=0,84)$. Para fines de este trabajo se ofrecieron también siete opciones de respuesta que iban desde estar "Totalmente de acuerdo" hasta "Totalmente en desacuerdo" con cada afirmación.

\section{Cuestionario de información sociodemográfica}

Por medio de un cuestionario elaborado ad hoc se indagaron características sociodemográficas. Se consultó por el sexo, el cantón de procedencia (zona provincial específica en donde habitaba el participante), la edad en años y meses, el tipo 
de secundaria de la cual se graduó el estudiante (secundarias diurnas académicas, nocturnas o de bachillerato por madurez) y el tipo de familia en la cual creció el participante (clasificada dependiendo de la presencia, cercanía o completa ausencia de ambos padres biológicos).

Además, se consultó por la cantidad de hijos; cantidad de personas que conviven en la misma casa; cantidad de hermanos en total; cantidad de hermanos mayores; cantidad de hermanos menores; nivel económico (consultado por medio de la existencia de casa propia en la familia, existencia de una habitación para cada persona, automóvil propio, televisión por cable, acceso a Internet, agua caliente en toda la casa, y cantidad de celulares y autos en la casa); el nivel educativo del padre y la madre, y la cantidad de horas semanales dedicadas a trabajos no relacionados con los estudios.

Como se señaló, estas variables fueron incluidas con un propósito básicamente exploratorio, ya que no han sido incluidas en la literatura previa. Se procuró incluir esta cantidad extensa de variables sociodemográficas para poseer un amplio espectro de opciones para indagar de modo inductivo posibles asociaciones significativas.

\section{Procedimiento}

\section{Traducción de la escala}

Un primer paso en la elaboración del estudio fue la traducción del SRSI-SR. Para desarrollar tal procedimiento se aplicó un método abreviado de traducción inversa como el recomendado por Cha, Kim y Erlen (2007) que sigue un proceso iterativo de tres fases: a) tres hablantes bilingües traducen los ítems del idioma original al idioma objetivo; b) un hablante bilingüe independiente traduce los ítems de forma inversa del idioma objetivo al idioma original sin conocer los ítems originales; c) un hablante nativo del idioma original compara los ítems originales con los traducidos a la inversa. En los casos en que no se encuentre equivalencia en los ítems originales y los traducidos se repite el proceso hasta alcanzar una traducción satisfactoria.

\section{Diseño de sitio web}

Como paso siguiente se incluyeron los instrumentos del estudio en un sitio web para la recolección automatizada de los datos. En el sitio se escribió un párrafo para presentar el estudio y asegurar sus requisitos éticos (consentimiento, participación voluntaria no remunerada y absoluta confidencialidad de la información). Luego se presentaron los instrumentos en el siguiente orden: 1) cuestionario de autorregulación general, 2) SRSI-SR y 3) cuestionario de información sociodemográfica.

\section{Recolección de datos}

Se visitaron grupos de carreras universitarias en un centro de educación superior de San José, Costa Rica. Los grupos de estudiantes fueron llevados a laboratorios de informática en donde se les facilitaba el enlace del sitio web que incluía los instrumentos. Cada estudiante completó los cuestionarios de modo personal.

\section{Sistematización de datos}

Los datos recolectados desde el sitio web fueron descargados en un archivo de Excel. Este archivo se editó y se exportó a un archivo de PSPP y a un archivo de block de notas para prepararlo para el análisis. Dichos archivos poseen las características propias de las matrices de datos, incluyendo las variables en columnas y los casos en filas.

\section{Análisis de datos}

\section{Análisis factorial exploratorio (AFE) $y$ análisis de fiabilidad}

Se realizaron la prueba de esfericidad de Bartlett y la prueba KMO para observar si existían 
suficientes relaciones entre los ítems. El AFE propiamente dicho se ejecutó siguiendo un método de rango mínimo (Shapiro \& Ten Berge, 2002), una rotación oblicua Promin (Lorenzo-Seva, 2013). Para determinar el número de factores se realizó un análisis paralelo a partir de una implementación óptima, el cual consiste en la comparación del gráfico de sedimentación con los autovalores que se obtendrían si los datos no presentaran factores comunes (Ledesma \& Valero-Mora, 2007; Timmerman \& Lorenzo-Seva, 2011).

Para cada dimensión obtenida en el AFE, así como para la medición general del AAR, se obtuvo el coeficiente de fiabilidad alfa de Cronbach. Los procedimientos del AFE se ejecutaron por medio del programa de uso libre Factor 9.3 (Baglin, 2014), y el análisis de fiabilidad se realizó por medio tanto del programa Factor (en su versión factorial) como del programa PSPP (GNU, 2014), también de uso libre. Para el AFE y para el cálculo de la fiabilidad y la obtención de la medición general de AAR, los ítems que hipotéticamente debían saturar en la subescala CRM fueron codificados de forma inversa. Para los otros análisis dichos ítems se mantuvieron con su codificación original.

\section{Análisis descriptivos y prueba de normalidad}

Se calcularon los estadísticos descriptivos para la medición general de AAR y para sus dimensiones identificadas por medio del AFE. Se obtuvieron la media, el rango, la desviación típica, el coeficiente de variación, la asimetría y su error típico, así como curtosis y su error típico. Se aplicó la prueba Kolmogorov-Smirnov (KS) para probar la distribución normal de la medición general del AAR y de sus dimensiones. Estos análisis descriptivos y los análisis que se describen a continuación se ejecutaron por medio del programa PSPP, a excepción de cuando se indica que se usó otro software.

\section{Análisis correlacionales}

Se obtuvo la correlación de Pearson entre la medición del ARG y la medición general del AAR, así como las correlaciones entre la medición del ARG y las dimensiones identificadas por medio del AFE. Se esperó obtener asociaciones significativas en estos análisis como evidencia preliminar de validez concurrente. Como medida de tamaño del efecto se obtuvo además el coeficiente de determinación $\left(\mathrm{R}^{2}\right)$.

\section{Análisis de la relación de dimensiones de AAR $y$ variables sociodemográficas}

Se exploró si alguna variable sociodemográfica establecía relaciones significativas con la medición general del AAR o con sus dimensiones. Se realizaron pruebas $\mathrm{T}$ para muestras independientes al analizar las diferencias entre sexos, Anova de una vía para las variables de tipo secundaria (secundarias diurnas académicas, diurnas técnicas y nocturnas o de bachillerato por madurez), tipo de familia (familias con ambos padres biológicos presentes, familias con sólo la madre biológica presente en contacto con el padre biológico y familias con sólo la madre biológica presente sin contacto con el padre biológico), y nivel educativo del padre y la madre (primaria incompleta o completa, secundaria incompleta o completa, universitaria incompleta o completa).

Se obtuvieron correlaciones no paramétricas con las variables edad, cantidad de hijos, cantidad de personas que conviven en el mismo hogar, cantidad de hermanos en total, cantidad de hermanos mayores y menores, nivel económico y cantidad de horas semanales dedicadas a trabajos no relacionados con los estudios. Fueron tomadas en cuenta aquellas relaciones significativas que fueron consistentes a través de las diferentes dimensiones del AAR como en el AAR general; además, se informa acerca de otras relaciones significativas pero se aclara cuándo no fueron consistentes. Como se 
mencionó, por medio del análisis se exploró inductivamente si alguna variable sociodemográfica se relacionaba con el autoinforme del AAR.

Se utilizó el programa $\mathrm{R}$ (R Development Core Team, 2012) para realizar las pruebas post hoc de Tukey y las correlaciones no paramétricas, así como para obtener los estadísticos $d$ de Cohen para las pruebas t y eta cuadrado para los Anova.

\section{Resultados}

La prueba de esfericidad resultó significativa (2734,26, $\mathrm{gl}=378, \mathrm{p}=0,0001)$, y el índice KMO alcanzó un valor de 0,835 . Esto mostró la existencia de suficientes relaciones para aplicar el AFE. El análisis paralelo estableció que debían mantenerse tres dimensiones, las cuales concentraron un $40,31 \%$ de la varianza acumulada. La tabla 1 muestra la matriz rotada que indica cuáles ítems saturaron en cada dimensión. Siguiendo los resultados de Cleary (2006), el primer factor representó la dimensión BAI, el segundo fue la dimensión CRM $\mathrm{y}$ el tercero fue la dimensión MAC.

Los ítems 3, 21 y 27 alcanzaron saturaciones de al menos 0,3 en al menos dos dimensiones, mientras que los ítems 11 y 18 no alcanzaron saturaciones mayores a 0,3 en ninguna dimensión. El ítem 24 tuvo una saturación no esperada dado que originalmente es parte de la dimensión MAC, pero en este análisis saturó en la dimensión BAI. Estos ítems fueron retirados de procedimientos siguientes a causa de estas ambigüedades. La subescala BAI quedó compuesta por seis ítems, en la subescala de CRM se mantuvieron siete ítems y en la subescala de MAC se mantuvieron nueve ítems.

En la tabla 2 se presentan el índice de fiabilidad alfa, los estadísticos descriptivos y el resultado de la prueba de normalidad para las dimensiones es-

Tabla 1

Análisis factorial exploratorio del SRSI-SR

\begin{tabular}{|c|c|c|c|}
\hline Ítem & Factor 1 & Factor 2 & Factor 3 \\
\hline 1. Me aseguro que nadie me moleste cuando estudio & 0,076 & 0,073 & 0,598 \\
\hline 2. Trato de estudiar en un lugar tranquilo & 0,125 & 0,031 & 0,689 \\
\hline 3. Pienso sobre los tipos de preguntas que podrían hacerme en un examen & 0,419 & 0,112 & 0,381 \\
\hline 4. Consulto al profesor sobre los temas que podrían preguntarse en los exámenes & 0,369 & $-0,027$ & 0,156 \\
\hline 5. Confío en mis notas de clase para estudiar & 0,305 & $-0,029$ & 0,134 \\
\hline 6. Estudio duro incluso cuando hay cosas más divertidas que hacer & $-0,026$ & $-0,134$ & 0,578 \\
\hline 7. Me hago exámenes yo mismo para ver qué tanto estoy aprendiendo mientras estudio & $-0,075$ & 0,044 & 0,592 \\
\hline 8. Hago un horario para ayudarme a organizar mi tiempo de estudio & $-0,142$ & 0,082 & 0,642 \\
\hline 9. Uso folders para organizar mis materiales de estudio & 0,171 & $-0,037$ & 0,443 \\
\hline 10. He perdido importantes materiales de mis clases $(\mathrm{R})$ & 0,034 & 0,607 & $-0,054$ \\
\hline 11. Evito ir a buscar clases particulares extra de mis materias $(\mathrm{R})$ & 0,095 & 0,153 & $-0,066$ \\
\hline 12. Espero hasta el último minuto para estudiar para los exámenes $(\mathrm{R})$ & 0,164 & 0,336 & $-0,509$ \\
\hline 13. Trato de olvidar aquellos temas sobre los cuales he tenido problemas al aprenderlos (R) & $-0,085$ & 0,600 & 0,046 \\
\hline 14. Trato de ver cómo mis notas de clase se relacionan con cosas que ya sé & 0,709 & $-0,018$ & $-0,233$ \\
\hline 15. Trato de identificar el formato de los próximos exámenes & 0,641 & 0,212 & $-0,152$ \\
\hline $\begin{array}{l}\text { 16. Trato de estudiar en un lugar que no tenga distracciones (por ejemplo, ruido, gente } \\
\text { hablando) }\end{array}$ & 0,187 & 0,086 & 0,623 \\
\hline
\end{tabular}




\begin{tabular}{|c|c|c|c|}
\hline Ítem & Factor 1 & Factor 2 & Factor 3 \\
\hline 17. Pregunto a mi profesor cuando no entiendo algo & 0,622 & $-0,261$ & 0,068 \\
\hline 18. Hago imágenes o dibujos para ayudarme a aprender conceptos & 0,280 & 0,027 & 0,251 \\
\hline 19. Me rindo o me doy por vencido cuando no entiendo algo (R) & $-0,005$ & 0,740 & 0,026 \\
\hline 20. Olvido llevar a casa los materiales cuando necesito estudiar (R) & 0,064 & 0,672 & 0,006 \\
\hline 21. Me digo a mí mismo exactamente lo que quiero lograr mientras estudio & 0,456 & 0,128 & 0,314 \\
\hline 22. Miro las notas de mis tareas si no entiendo algo & 0,477 & 0,002 & 0,097 \\
\hline 23. Evito hacer preguntas en clase sobre cosas que no entiendo (R) & $-0,230$ & 0,691 & 0,238 \\
\hline $\begin{array}{l}\text { 24. Me digo a mí mismo que hay que seguir tratando cuando no puedo entender un tema } \\
\text { o idea }\end{array}$ & 0,533 & $-0,124$ & 0,145 \\
\hline 25. Organizo cuidadosamente mis materiales de estudio de modo que no los pierdo & 0,294 & $-0,143$ & 0,456 \\
\hline 26. Dejo a mis amigos interrumpirme cuando estoy estudiando $(\mathrm{R})$ & 0,259 & 0,446 & $-0,354$ \\
\hline 27. Pienso acerca de cuál es el mejor modo de estudiar antes de empezar a estudiar & 0,387 & 0,096 & 0,365 \\
\hline 28. Termino todo lo que tengo que estudiar antes de jugar videojuegos o con mis amigos & $-0,040$ & $-0,092$ & 0,520 \\
\hline Autovalor & 6,893 & 2,513 & 1,880 \\
\hline Porcentaje de varianza & 24,62 & 8,98 & 6,71 \\
\hline
\end{tabular}

$\mathrm{R}$ : Indica un ítem que fue codificado inversamente.

La carga presentada en cursiva indica que pertenece al factor representado en cada columna.

pecíficas obtenidas en el AFE y para la medición general del AAR. Como puede observarse, las fiabilidades de todas las dimensiones han sido aceptables o buenas. Según la prueba KS, solamente la dimensión MAC se ha desviado de la normalidad, presentando una clara tendencia leptocúrtica y un sesgo asimétrico negativo.
En la tabla 3 se presentan las correlaciones entre la medición del ARG, las dimensiones específicas y la medición general del AAR. Todas las asociaciones fueron significativas, tanto las relaciones entre elARG y BAI como entre el ARG y el AAR general llegaron a ser moderadas; la asociación entre el ARG y MAC y la relación negativa entre el ARG

Tabla 2

Fiabilidad, estadisticos descriptivos y prueba de normalidad para dimensiones especificas y medición general del AAR

\begin{tabular}{|c|c|c|c|c|c|c|c|c|}
\hline Dimensión & $\alpha$ & $\mathrm{M}$ & $\mathrm{R}$ & DT & $\mathrm{CV}$ & $\begin{array}{l}\text { Asim. } \\
\text { (e.t.) }\end{array}$ & $\begin{array}{l}\text { Curt. } \\
\text { (e.t.) }\end{array}$ & $\mathrm{Z}$ \\
\hline BAI & $\begin{array}{l}0,710 \\
0,834\end{array}$ & 31,41 & 33 & 5,40 & $17,19 \%$ & $\begin{array}{l}-0,395 \\
(0,145)\end{array}$ & $\begin{array}{c}1,062 \\
(0,289)\end{array}$ & $\begin{array}{c}0,955(\mathrm{p}< \\
0,321)\end{array}$ \\
\hline CRM & $\begin{array}{l}0,766 \\
0,837\end{array}$ & 19,06 & 42 & 6,93 & $36,36 \%$ & $\begin{array}{c}0,467 \\
(0,145)\end{array}$ & $\begin{array}{c}1,035 \\
(0,289)\end{array}$ & $\begin{array}{c}1,056(\mathrm{p}< \\
0,215)\end{array}$ \\
\hline MAC & $\begin{array}{l}0,815 \\
0,869\end{array}$ & 44,98 & 54 & 9,03 & $20,08 \%$ & $\begin{array}{c}-0,951 \\
(0,145)\end{array}$ & $\begin{array}{c}2,129 \\
(0,289)\end{array}$ & $\begin{array}{c}1,384(\mathrm{p}< \\
0,043)\end{array}$ \\
\hline AAR General & $\begin{array}{l}0,847 \\
0,873\end{array}$ & 113,33 & 114 & 16,44 & $14,51 \%$ & $\begin{array}{c}-0,501 \\
(0,145)\end{array}$ & $\begin{array}{c}1,214 \\
(0,289)\end{array}$ & $\begin{array}{c}0,778(\mathrm{p}< \\
0,580)\end{array}$ \\
\hline
\end{tabular}

$\alpha$ : Coeficiente alfa obtenido en PSPP, en cursiva se presenta el coeficiente alfa obtenido en su versión factorial en Factor 9.3, M: Media, R: Rango, DT: Desviación típica, CV: Coeficiente de variación, Asim.: Asimetría, Curt.: Curtosis, e.t.: error típico, $Z=$ valor $Z$ de prueba KS 
y CRM fueron leves. Los coeficientes de determinación muestran tamaños de efectos pequeños.

Tabla 3

Correlaciones de orden cero y coeficiente de determinación entre el ARG, las dimensiones especificas del AAR y el AAR general

\begin{tabular}{ccccc}
\hline & BAI & CRM & MAC & AAR general \\
\hline ARG r & $0,391^{* *}$ & $-0,147^{*}$ & $0,287^{* *}$ & $0,348^{* *}$ \\
$\mathrm{R}^{2}$ & 0,153 & 0,022 & 0,082 & 0,121 \\
\hline
\end{tabular}

**Correlación significativa para $\mathrm{p}<0,01$

*Correlación significativa para $\mathrm{p}<0,05$

Como se muestra en la tabla 4, al considerar las diferentes variables sociodemográficas se encontró un efecto del sexo sobre las dimensiones BAI, MAC y en la medida general del AAR; no hubo diferencias significativas según sexo en CRM. En todas las diferencias significativas las medias femeninas $(n=149)$ fueron superiores a las masculinas ( $n=134)$, el estadístico d de Cohen muestra un tamaño del efecto leve en todos los casos. La figura 1 presenta estos resultados.
Tabla 4

Diferencias de medias según sexo en dimensiones del AAR y el AAR general

\begin{tabular}{|c|c|c|c|c|}
\hline $\begin{array}{l}\text { Medias según } \\
\text { sexo }\end{array}$ & $\mathrm{t}(\mathrm{gl})$ & p. & $\begin{array}{l}\text { Diferencia } \\
\text { entre } \\
\text { medias }\end{array}$ & $\begin{array}{c}d . \\
\text { L.I. }- \text { L.S }\end{array}$ \\
\hline $\begin{array}{l}\text { BAI } \\
\text { Hombres: } 30,71 \\
\text { Mujeres: } 32,04\end{array}$ & $\begin{array}{l}-2,084 \\
(281)\end{array}$ & 0,038 & $-1,33$ & $\begin{array}{c}-0,25 \\
-1,11- \\
0,64\end{array}$ \\
\hline $\begin{array}{l}\text { CRM } \\
\text { Hombres: } 19,72 \\
\text { Mujeres: } 18,46\end{array}$ & $\begin{array}{l}1,531 \\
(281)\end{array}$ & 0,127 & 1,26 & $\begin{array}{c}0,18 \\
-1,04- \\
1,23\end{array}$ \\
\hline $\begin{array}{l}\text { MAC } \\
\text { Hombres: 43,38 } \\
\text { Mujeres: } 46,41\end{array}$ & $\begin{array}{l}-2,854 \\
(281)\end{array}$ & 0,005 & $-3,03$ & $\begin{array}{r}-0,34 \\
-1,94- \\
0,99\end{array}$ \\
\hline $\begin{array}{l}\text { AAR general } \\
\text { Hombres: } 110,37 \\
\text { Mujeres: } 115,99\end{array}$ & $\begin{array}{l}-2,910 \\
(281)\end{array}$ & 0,004 & $-5,62$ & $\begin{array}{c}-0,34 \\
-3,17- \\
2,18\end{array}$ \\
\hline
\end{tabular}

L.I.: Límite inferior. L.S.: Límite superior

La otra variable sociodemográfica que estableció relaciones relativamente más consistentes con las diferentes dimensiones del AAR y el AAR general fue el tipo de familia del estudiante. Re-

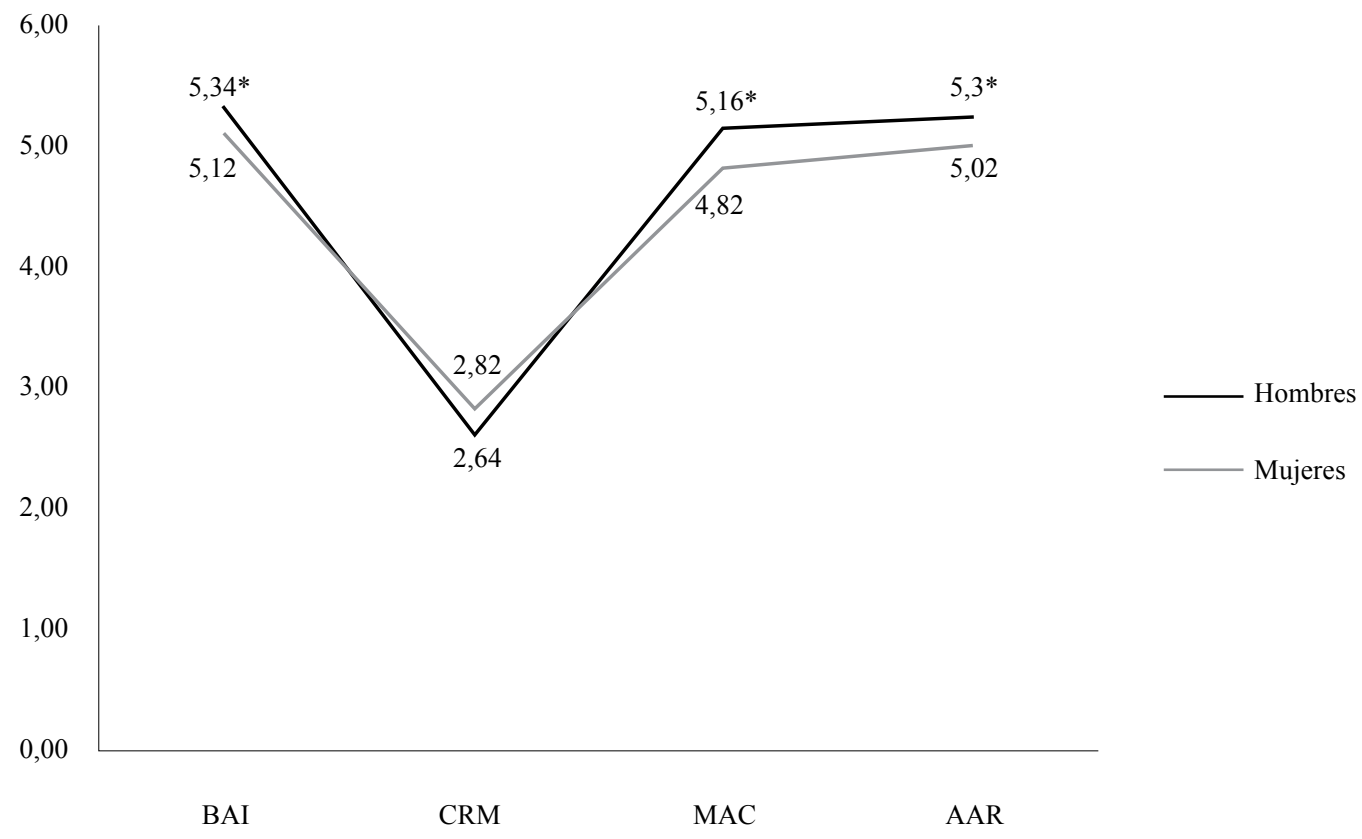

Figura 1. Medias según sexo en las dimensiones del AAR y el AAR general

* Diferencias significativas. Las medias se presentan divididas entre la cantidad de ítems de cada subescala. 
cuérdese que se tomaron en cuenta tres tipos de familias: dos padres biológicos presentes $(n=203)$, sólo la madre biológica presente pero el padre biológico se ha mantenido en contacto $(\mathrm{n}=38)$ y sólo la madre biológica presente con el padre biológico totalmente ausente $(\mathrm{n}=31)$. Aunque se consultó sobre otros tipos de familia (padres biológicos presentes pero madres ausentes, así como padre y madre biológicos ausentes) la frecuencia absoluta correspondiente a estos otros tipos de familias resultó demasiado escasa como para poder aplicar cualquier tipo de análisis.

La tabla 5 muestra los resultados del Anova de una vía tomando en cuenta el tipo de familia como factor y las diferentes dimensiones del AAR y el AAR general como variables dependientes. Tal y como puede observarse, los tamaños de efecto han sido en general pequeños, a excepción del tamaño del efecto encontrado al considerar el AAR general, que puede considerarse mediano.

Las pruebas post hoc de Tukey mostraron que al considerar la dimensión BAI las familias con sólo la madre presente y el padre manteniendo contacto $(\mathrm{M}=33,39$; DT $=4,79)$ puntuaron significativamente más alto que las familias con presencia de ambos padres biológicos $(\mathrm{M}=30,93$; DT $=5,61)$ $(\mathrm{p}=0,026) ;$ no existió otra diferencia significativa entre grupos en esta dimensión. Al tomar en cuenta las CRM, la prueba post hoc no evidenció ninguna diferencia significativa entre grupos, por lo que el efecto significativo encontrado en el Anova no apareció al hacer las comparaciones posteriores entre grupos.

En la dimensión MAC existieron diferencias significativas entre las familias con los dos padres biológicos presentes $(\mathrm{M}=43,60 ; \mathrm{DT}=9,11) \mathrm{y}$ las familias con sólo la madre presente y el padre manteniendo contacto $(\mathrm{M}=48,81 ; \mathrm{DT}=7,44)(\mathrm{p}$ $=0,003)$, así como entre las familias con ambos padres presentes y las familias con sólo la madre presente y el padre totalmente ausente $(\mathrm{M}=47,77$; $\mathrm{DT}=8,12)(\mathrm{p}=0,003)$. Como puede observarse, las medias mayores siempre estuvieron en las familias monoparentales maternas.

Un fenómeno similar al anterior ocurrió al considerar la medida del AAR general. Las familias biparentales biológicas $(\mathrm{M}=110,83 \mathrm{DT}=16,34)$ puntuaron menos que las familias monoparentales maternas con contacto con el padre $(\mathrm{M}=120,92$; $\mathrm{DT}=14,98)(\mathrm{p}=0,001)$ y que las familias monoparentales sin contacto con el padre $(M=119,19$; $\mathrm{DT}=13,63)(\mathrm{p}=0,018)$. Como puede constatarse,

Tabla 5

ANOVA de una vía. Variables dependientes: dimensiones del AAR y el AAR general. Factor: tipo de familia

\begin{tabular}{lcccccc}
\hline \multicolumn{1}{c}{ Variable } & $\begin{array}{c}\text { Suma de } \\
\text { cuadrados }\end{array}$ & g.l. & $\begin{array}{c}\text { Cuadrado } \\
\text { medio }\end{array}$ & F & p. & $\eta^{2}$ \\
\hline BAI Entre grupos & 213,748 & 2 & 106,874 & 3,755 & 0,025 & 0,027 \\
Intra grupos & 7656,307 & 269 & 28,462 & & & \\
Total & 7870,055 & 271 & & & & \\
\hline CRM Entre grupos & 369,747 & 2 & 184,874 & 3,916 & 0,021 & 0,028 \\
Intra grupos & 12698,017 & 269 & 47,205 & & & \\
Total & 13067,765 & 271 & & & & \\
\hline MAC Entre grupos & 1178,863 & 2 & 589,432 & 7,624 & 0,001 & 0,054 \\
Intra grupos & 20797,810 & 269 & 77,315 & & & \\
Total & 21976,673 & 271 & & & & \\
\hline AAR Entre grupos & 4521,491 & 2 & 2260,746 & 8,968 & 0,001 & 0,063 \\
General Intra grupos & 67812,567 & 269 & 252,091 & & & \\
Total & 72334,059 & 271 & & & & \\
\hline
\end{tabular}


a excepción de la dimensión CRM, siempre existió alguna diferencia entre familias biparentales y uno o ambos tipos de familias monoparentales maternas tanto al considerar el AAR general o sus dimensiones particulares.

Otras variables sociodemográficas establecieron relaciones significativas aunque no tuvieron la consistencia de las presentadas antes. Se encontró, por ejemplo, un efecto del tipo de secundaria en la dimensión BAI $(\mathrm{F}(282)=3,512, \mathrm{p}=0,031)$, los estudiantes de secundarias diurnas académicas $(M$ $=31,80$; D.T. $=5,29)$ puntuaron más alto que los de diurna técnica $(M=29,45 ; \mathrm{DT}=5,38)$.

Así también el nivel educativo de la madre estableció diferencias significativas en BAI (F (282) $=3,631, p=0,028)$. Los estudiantes de madres con educación universitaria incompleta o completa puntuaron significativamente más $(\mathrm{M}=32,40$; $\mathrm{DT}=5,35)$ que los estudiantes de madres con sólo educación primaria incompleta o completa $(\mathrm{M}=$ $30,11 ; \mathrm{DT}=5,43)$. El nivel educativo del padre no tuvo ningún efecto significativo.
La dimensión BAI estableció otras relaciones significativas, estuvo negativamente asociada con la cantidad de hermanos $(\rho=-0,172 ; p=0,004)$ y con la cantidad de hermanos mayores $(\rho=-0,169$; $\mathrm{p}=0,004)$. Así también la medición general del AAR se asoció negativamente con la cantidad de hermanos mayores $(\rho=-0,142 ; p=0,017)$. No hubo otras asociaciones significativas.

\section{Discusión}

El presente estudio ha desarrollado una adaptación en español del SRSI-SR, instrumento que busca evaluar el autoinforme sobre el AAR que hace el estudiante. A pesar de que el trabajo ha tenido diferencias metodológicas y se ha desarrollado con estudiantes de un nivel académico diferente al estudio que originalmente creó el instrumento (Cleary, 2006), los resultados han sido casi idénticos. La estructura factorial esperada se ha replicado en este trabajo, solamente ha sido necesario retirar seis ítems que no saturaron en ninguna dimensión,

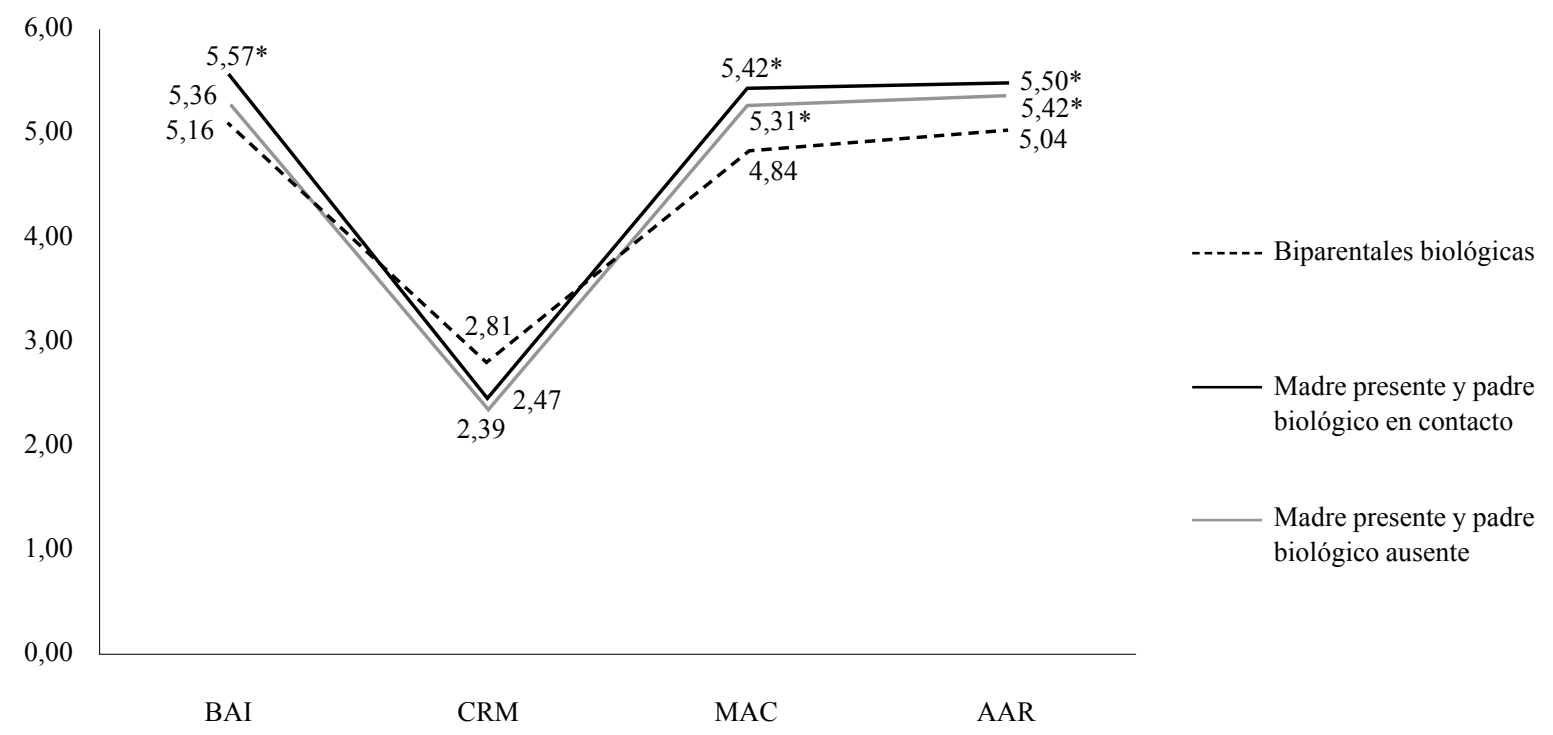

Figura 2. Medias según tipo de familia en dimensiones del AAR y el AAR general.*Diferencias significativamente superiores con respecto a las familias biparentales biológicas. Las medias se presentan divididas entre la cantidad de ítems de cada subescala. 
tuvieron cargas factoriales ambiguas o bien saturaron en una dimensión diferente a la esperada. Esto ha resultado en un instrumento de 22 ítems con tres dimensiones claramente delineadas: "búsqueda de aprendizaje e información”, "comportamientos regulatorios maladaptativos" y "manejo del ambiente y el comportamiento".

Tanto los coeficientes de consistencia interna de las diferentes subescalas y de la medición general, como las correlaciones significativas de éstas con una medición independiente del ARG son muestra de que el trabajo de validación va por buen camino. Futuros estudios deben probar los distintos tipos de validez y aplicar análisis factoriales confirmatorios para robustecer la evidencia empírica de la calidad del instrumento.

En lo relacionado con las características descriptivas de las variables identificadas por medio del instrumento, llama la atención cómo la dimensión CRM presentó un coeficiente de variación marcadamente más alto que las otras dimensiones y también mayor que la medición del AAR general. En el trabajo antecedente inmediato (Cleary, 2006) los hallazgos obtenidos difieren bastante de estos resultados, dado que se presentó el fenómeno contrario: el coeficiente de variación de la dimensión CRM, estimado a partir de las medias y desviaciones típicas, fue comparativamente menor que el de las otras dimensiones en todas las distintas submuestras estudiadas por el autor divididas por sexo y nivel académico.

Por otro lado, en el trabajo de Cleary y Chen (2009) los coeficientes de variación también fueron menores en comparación a la otra subescala de AAR utilizada en todas las submuestras estudiadas sin importar el nivel académico y el nivel de logro. Esto muestra que posiblemente factores como el tipo de contexto académico o la evaluación del AAR de modo general o dirigido a un dominio temático afectan la variabilidad de las dimensiones.

Futuros trabajos deberán considerar además si resultados como el anterior se replican. Las variaciones entre diferentes estudios se pueden deber a diferencias en los contextos culturales y educativos o a las diferencias metodológicas entre distintos estudios. Recuérdese, por ejemplo, que mientras aquí se ha solicitado a los estudiantes que informen de sus características del AAR sin hacer referencia a ningún contexto en específico, los estudios previos solicitaban a los estudiantes que se evaluaran haciendo referencia a un contexto académico específico (por ejemplo, sus capacidad del AAR en ciencias o matemáticas).

Así mismo, mientras que los estudios de Cleary (2006) y Cleary y Chen (2009) han sido realizados con muestras de estudiantes de secundaria, el presente trabajo se desarrolló con estudiantes universitarios. La posible existencia de diferencias en el autoinforme del AAR dependientes de evaluaciones específicas según el contexto académico o el tipo de materia estudiada, y las posibles diferencias culturales o de nivel académico (secundaria vs. estudios universitarios) podrían relacionarse con las características disímiles encontradas entre diferentes muestras.

Un resultado que sí es más estable entre los diferentes estudios es la relación entre el sexo del estudiante y el nivel del AAR. Tanto Cleary (2006) como Cleary y Chen (2009) encontraron mayores niveles de uso de estrategias de autorregulación entre las mujeres en comparación a los varones; a pesar de las diferencias metodológicas y del marco muestral ya referido, el resultado ha sido el mismo en el presente trabajo: las mujeres han puntuado significativamente más en el autoinforme de estrategias positivas de autorregulación del aprendizaje. Sin embargo, debe tenerse en cuenta que el tamaño del efecto obtenido ha sido leve, por lo cual este tipo de resultados deben considerarse en su justa dimensión y no sobreestimar el efecto del sexo por encima de otras variables que deberán ser evaluadas después.

No obstante, se torna interesante considerar a qué podría deberse el efecto del sexo tanto en este estudio como en los trabajos previos que han utilizado el SRSI-SR. Una posibilidad es que las 
diferencias de género encontradas sean un fenómeno dependiente de la medición o de la metodología utilizada. Por ejemplo, utilizando otro tipo de metodologías e instrumentos estudios como los realizados por Yukselturk y Bulut (2009) y Hong, Peng y Rowell (2009) no encontraron diferencias significativas entre medias del AAR entre géneros. Sin embargo, en la mayor parte de los trabajos previos sí se informa de diferencias entre géneros en el uso de estrategias de regulación del aprendizaje, y aunque en algunos casos no se ha evaluado si las mujeres informan de un mayor nivel de AAR (Lee, 2002), típicamente los hallazgos concuerdan en que las mujeres puntúan más en el AAR en comparación con los hombres (Bidjerano, 2005; Matthews, Marulis, y Williford; 2014; Pajares, 2002).

No solamente en el tema del AAR parece existir esta diferencia de género consistente, sino que las capacidades autorregulatorias generales son distintas entre géneros y tal diferencia tiende a establecerse en tempranos años de la vida. Por ejemplo, Matthews, Cameron y Morrison (2009) encontraron que ya entre niños preescolares las mujeres puntuaban más alto que los varones en tareas de autorregulación y en la evaluación que hacían los maestros de tal variable al valorar a sus estudiantes; no obstante, a estas tempranas edades las diferencias en autorregulación no llevan a diferencias de género en el logro académico. Evidencia adicional sugiere que estas tempranas diferencias de género en procesos autorregulatorios pueden ser culturalmente dependientes (véase, por ejemplo, Wanless et al., 2013).

La relación encontrada con el tipo de familia también ha sido una asociación leve. Hasta el momento la medición del AAR tomada por medio del SISR-SR no había sido relacionada con dicha variable, no obstante fenómenos psicológicos de autorregulación se han relacionado previamente con las relaciones padres-hijos en adolescentes (véase, por ejemplo, Farley \& Kim-Spoon, 2014) y la estructura familiar. También en dicho periodo del curso vital, aunque contrario al resultado del presente estudio, la monoparentalidad se ha relacionado con déficits en autorregulación (Nobile et al., 2014).

En general el tema de la relación de procesos y características de la familia con fenómenos psicológicos autorregulatorios ha sido más investigado en la infancia (Grolnick, Kurowski, \& Gurland, 1999; Grolnick \& Farkas, 2002; Kopp, 1982) y no en adultos jóvenes, como ha sido el caso de la presente investigación. En este trabajo la asociación entre el tipo de familia y las dimensiones del AAR parece bastante estable ya que ha ocurrido en dos dimensiones particulares del AAR y en la medición de AAR general. No obstante, este resultado debe ser replicado y hasta entonces debe ser tratado con cautela.

Las relaciones de las dimensiones del AAR con otras variables sociodemográficas no resultan tan consistentes como las encontradas con el sexo y el tipo de familia, por lo que probablemente se trate de correlaciones espurias; éstas se reportan para dar una descripción completa de las asociaciones significativas encontradas.

\section{Referencias}

Babakhani, N. (2014). The relationship between the big-five model of personality, self-regulated learning strategies and academic performance of Islamic Azad University Students. Procedia - Social and Behavioral Sciences, 116, 3 5423 547. doi: 10.1016/j.sbspro.2014.01.799

Bandura, A. (1997). Self-efficacy: The exercise of control. Nueva York: W.H. Freeman.

Bidjerano, T. (2005). Gender differences in self-regulated learning. Annual Meeting of the Northeastern Educational Research Association, October 19-21, Kerhonkson, NY.

Baglin, J. (2014). Improving your exploratory factor analysis for ordinal data: A demonstration using FACTOR. Practical Assessment, Research \& Evaluation, 19, 2-11. 
Boekaerts, M. \& Corno, L. (2005). Self-regulation in the clasroom: a perspective on assessment and intervention. Applied Psychology: An International Review, 54, 199-231. doi: 10.1111/j.14640597.2005.00205.x

Boekaerts, M., Pintrich, P. R., \& Zeidner, M. (Eds.) (2000). Self-regulation: Theory, research, and applications. Orlando, FL: Academic Press.

Cazan, A. M. (2012). Self-regulated learning-strategies - predictors of academic adjustment. Procedia - Social and Behavioral Sciences, 33, 104-108. doi: 10.1016/j.sbspro.2012.01.092

Cha, E. S., Kim, K., \& Erlen, J. (2007). Translation of scales in cross-cultural research: issues and techniques. Journal of Advanced Nursing, 58, 386395. doi: 10.1111/j.1365-2648.2007.04242.x

Clarebout, G., Horz, H., Schnotz, W., \& Elen, J. (2010). The relations between self-regulation and the embedding of support in learning environments. Educational Technology Research and Development, 58, 573-587. doi: 10.1007/ s11423-009-9147-4

Cleary, T. J. (2006). The development and validation of the Self-Regulation Strategy Inventory-Self-Report. Journal of School Psychology, 44, 307-322. doi: 10.1016/j.jsp.2006.05.002

Cleary, T. J. \& Chen, P. P. (2009). Self-regulation, motivation, and math achievement in middle school: Variations across grade level and math context. Journal of School Psychology, 47, 291314. doi: 10.1016/j.jsp.2009.04.002

Cleary, T. J. \& Platten, P. (2013). Examining the correspondence between self-regulated learning and academic achievement: A case study analysis. Education Research International, 18 , 1-18. doi: 10.1155/2013/272560

de Bruin, A. B., Thiede, K. W., Camp, G., \& Redford, J. (2011). Generating keywords improves metacomprehension and self-regulation in elementary and middle school children. Journal of Experimental Child Psychology, 109, 294-310. doi: 10.1016/j.jecp.2011.02.005
Endedijk, M. D., Brekelmans, M., Verloop, N., Sleegers, P. J., \& Vermunt, J. D. (2014). Individual differences in student teachers' self-regulated learning: an examination of regulation configurations in relation to conceptions of learning to teach. Learning and Individual Differences, 30, 155-162. doi: 10.1016/j.lindif.2013.12.005

Farley, J. P. \& Kim-Spoon, J. (2014). The development of adolescent self-regulation: reviewing the role of parent, peer, friend, and romantic relationships. Journal of Adolescence, 37, 433440. doi: 10.1016/j.adolescence.2014.03.009

Friedrich, A., Jonkmann, K., Nagengast, B., Schmitz, B., \& Trautwein, U. (2013). Teachers' and students' perceptions of self-regulated learning and math competence: differentiation and agreement. Learning and Individual Differences, 27, 26-34. doi: 10.1016/j.lindif.2013.06.005

GNU (2014). PSPP Users' Guide. Release 0.8.5. Londres: GNU.

Greene, J. A., Hutchinson, L. A., Costa, L. J., \& Crompton, H. (2012). Investigating how collage students' task definitions and plans relate to self-regulated learning processing and understanding of a complex science topic. Contemporary Educational Psychology, 37, 307-320. doi: 10.1016/j.cedpsych.2012.02.002

Grolnick, W. S., Kurowski, C. O., \& Gurland, S. T. (1999). Family processes and the development of children's self-regulation. Educational Psychologist, 34, 3-14. doi: 10.1207/ s15326985ep3401_1

Grolnick W. S. \& Farkas, M. (2002). Parenting and the development of children's self-regulation. En M. Bornstein (Ed.), Handbook of Parenting. Volume 5: Practical Issues of Parenting (pp. 89109). New Jersey: Lawrence Erlbaum.

Hadi, M., Kalantari, B., \& Ghaslani, R. (2014). Self-regulated learning (SRL), motivation and language achievement of Iranian EFL learners. Procedia-Social and Behavioral Sciences, 98 , 1 062-1 068. doi: 10.1016/j.sbspro.2014.03.517 
Harris, K. R., Friedlander, B. D., Saddler, B., Frizzelle, R., \& Graham, S. (2005). Self-monitoring of attention versus self-monitoring of academic performance: Effects among students with ADHD in the general education classroom. Journal of Special Education, 39, 145-156. doi: 10.1177/00224669050390030201

Hong, E., Peng, Y., \& Rowell, L. L. (2009). Homework self-regulation: grade, gender, and achievement-level differences. Learning and Individual Differences, 19, 269-276. doi: 10.1016/j.lindif.2008.11.009

Jarvela, S. \& Jarvenoja, H. (2011). Socially constructed self-regulated learning and motivation regulation in collaborative learning groups. Teachers College Record, 113, 350-374.

Kindekens, A., Romero, V., De Backer, F., Peeters, J, Buffel, T., \& Lombaerts, K. (2014). Enhancing studen wellbeing in secondary education by combining self-regulated learning and arts education. Procedia - Social and Behavioral Sciences, 116, 1 982-1 987. doi: 10.1016/j.sbspro.2014.01.507

Kirk, K. M. \& Martin, N. G. (1998). The short interpersonal reactions inventory, self-regulation and differentiation scales in an older Australian twin sample. Personality and Individual Differences, 25, 591-604. doi: 10.1016/S01918869(98)00116-0

Kistner, S., Rakoczy, K., Otto, B., van Ewijk, C. D., Büttner, G., \& Klieme, E. (2010). Promotion of self-regulated learning in classrooms: Investigating frequency, quality, and consequences for student performance. Metacognition and Learning, 5, 157-171. doi: 10.1007/s11409-010-9055-3

Kolovelonis, A., Goudas, M., \& Dermitzaki, I. (2011). The effect of different goals and self-recording on self-regulation of learning a motor skill in a physical education setting. Learning and Instruction, 21, 355-364. doi: 10.1016/j.learninstruc.2010.04.001

Kopp, C. B. (1982). Antecedents of self-regulation: a developmental perspective. Developmental
Psychology, 18, 199-214. doi: 10.1037/00121649.18.2.199

Kuo, Y. C., Walker, A. E., Schroder, K. E., \& Belland, B. R. (2014). Interaction, Internet self-efficacy, and self-regulated learning as predictors of student satisfaction in online education courses. The Internet and Higher Education, 20, 35-50. doi: 10.1016/j.iheduc.2013.10.001

Labuhn, A. S., Zimmerman, B. J., \& Hasselhorn, M. (2010). Enhancing students' self-regulation and mathematics performance: The influence of feedback and self-evaluative standards. Metacognition and Learning, 5, 173-194. doi: 10.1007/s11409-010-9056-2

Ledesma. R. \& Valero-Mora, P. (2007). Determining the number of factors to retain in EFA: An essay to use computer program for carrying out $\mathrm{Pa}$ rallel Analysis. Practical Assesment Research and Evaluation, 12, 1-11.

Lee, I. S. (2002). Gender differences in self-regulated on-line learning strategies within Korea's university context. Educational Technology Research and Development, 50, 101-111. doi: 10.1007/BF02504967

Lehmann, T., Hähnlein, I., \& Ifenthaler, D. (2014). Cognitive, metacognitive and motivational prespectives on preflection in self-regulated online learning. Computers and Human Behavior, 32, 313-323. doi: 10.1016/j.chb.2013.07.051

Lorenzo-Seva, U. (2013). Why rotate my data using Promin? Technical report. Department of Psychology, Universitat Rovira i Virgil, Tarragona. Recuperado de http://psico.fcep.urv.es/utilitats factor/Documentation. html

Matthews, J. S., Cameron, C., \& Morrison, F. J. (2009). Early gender differences in self-regulation and academic achievement. Journal of Educational Psychology, 101, 689-704. doi: 10.1037/a0014240

Matthews, J. S., Marulis, L. M., \& Williford, A. P. (2014). Gender processes in school functioning and the mediating role of cognitive self-regulation. Journal of Applied Developmental 
Psychology, 35, 128-137. doi: 10.1016/j.appdev.2014.02.003

Nandagopal, K. \& Anders, K. (2012). An expert performance approach to the study of individual differences in self-regulated learning activities in upper-level collage students. Learning and Individual Differences, 22, 597-609. doi: 10.1016/j.lindif.2011.11.018

Nobile, M., Bianchi, M., Bellina, M., Greco, A., Monzani, D., Tesei, A., \& Molteni, M. (2014). EPA1746 - The role of family structure and of tryptophan hydroxylase 2 (TPH2) on the stability of deficient emotional self-regulation symptoms throughout adolescence. European Psychiatry, 29, 1. doi: 10.1016/S0924-9338(14)78877-7

Ozan, C., Gundogdu, K., Bay, E., \& Celkan, H. Y. (2012). A study on the University students' self-regulated learning strategie skills and self-efficacy perceptions in terms of different variables. Procedia - Social and Behavioral Sciences, 46, 1 806-1 811. doi: 10.1016/j.sbspro.2012.05.383

Pajares, F. (2002). Gender and perceived self-efficacy in self-regulated learning. Theory into Practice, 41, 116-125. doi: 10.1207/s15430421tip4102_8

Peeters, J., De Backer, F., Romero, V., Kindekens, A., Buffel, T., \& Lombaerts, K. (2014). The role of teachers' self-regulatory capacities in the implementation of self-regulated learning practices. Procedia-Social and Behavioral Sciences, 116, 1963-1 970. doi: 10.1016/j.sbspro.2014.01.504

Pintrich, P. R. (2003). A motivational science perspective on the role of student motivation in learning and teaching contexts. Journal of Educational Psychology, 95, 667-686. doi: 10.1037/00220663.95.4.667

Pintrich, P. R. \& Schunk, D. H. (2002). Motivation in education: Theory, research, and applications ( $2^{\mathrm{a}}$ ed.). Upper Saddle: Prentice-Hall, Inc.

R Development Core Team (2012). $R$ : a language and environment for statistical computing. Vienna: R Foundation for Statistical Computing.
Rosário, P., Pereira, A., Högemann, J., Nunes, A. R., Figueiredo, M., Núñez, J. C., Fuentes, S., \& Gaeta, M. L. (2013). Autorregulación del aprendizaje: una revisión sistemática en revistas de la base SciELO. Universitas Psychologica, 13, 781-798. doi: 10.11144/Javeriana. UPSY13-2.aars

Saks, K. \& Leijen, Ä. (2014). Distinguishing self-directed and self-regulated learning and measuring them in the e-learning context. ProcediaSocial and Behavioral Sciences, 112, 190-198. doi: 10.1016/j.sbspro.2014.01.1155

Schunk, D. H. (2005). Commentary on self-regulation in school contexts. Learning and Instruction, 15, 173-177. doi: 10.1016/j.learninstruc.2005.04.013

Schunk, D. \& Zimmerman, B. J. (2007). Influencing children's self-efficacy and self-regulation of reading and writing through modeling. Reading \& Writing Quarterly, 23, 7-25.

Shapiro, A. \& Ten Berge, J. M. F. (2002). Statistical inference of minimum rank factor analysis. Psychometrika, 67, 79-94. doi: 10.1007/ BF02294710

Taub, M., Azevedo, R., Bouchet, F., \& Khosravifar, B. (2014). Can the use of cognitive and metacognitive self-regulated learning strategies be predicted by learners' levels of prior knowledge in hypermedia-learning environments? Computers in Human Behavior, 39, 356-367. doi: 10.1016/j.chb.2014.07.018

Timmerman, M. E. y Lorenzo-Seva, U. (2011). Dimensionality assessment of ordered polytomous items with parallel analysis. Psychological Methods, 16, 209-220. doi: 10.1037/a0023353.

Vandevelde, S., Van Keer, H., \& Rosseel, Y. (2013). Measuring the complexity of upper primary school children's self-regulated learning: a multicomponent approach. Contemporary Educational Psychology, 38, 407-425. doi: 10.1016/j. cedpsych.2013.09.002

Wanless, S. B., McClelland, M. M., Lan, X., Son, S. H., Cameron, C. E., Morrison, F. J., Chen, F. M., 
Chen, J. L., Li, S., Lee, K., \& Sung, M. (2013). Gender differences in behavioral regulation in four societies: The United States, Taiwan, South Korea and China. Early Childhood Research Quarterly, 28, 621-633. doi: 10.1016/j. ecresq.2013.04.002

Wolters, C. A. (2011). Regulation of motivation: Contextual and social aspects. Teachers College Record, 113, 265-283.

Won You, J. \& Kang, M. (2014). The role of academic emotions in the relationship between perceived academic control and self-regulated learning in onlinelearning. Computers \& Education, 77, 125-133. doi: 10.1016/j.compedu.2014.04.018

Yukselturk, E. \& Bulut, S. (2009). Gender differences in self-regulated online learning environment. Education Technology \& Society, 12, 12-22.

Zimmerman, B. J. (1990). Self-regulated learning and academic achievement: an overview. Educational Psychologist, 25, 3-17. doi: 10.1207/ s15326985ep2501_2

Zimmerman, B. J. (2000). Attaining self-regulation: A social-cognitive perspective. En M. Boekaerts,
P. Pintrich, \& M. Zeidner (Eds.), Self-regulation: Theory, research, and applications (pp. 13-39). Orlando, FL: Academic Press.

Zimmerman, B. J. (2002). Achieving self-regulation: The trial and triumph of adolescence. En F. Pajares \& T. Urdan (Eds.), Academic motivation of adolescents, vol. 2 (pp. 1-27). Greenwhich: Information Age.

Zimmerman, B. J. (2008). Investigating self-regulation and motivation: Historical background, methodological developments, and future prospects. American Educational Research Journal, 45, 166-183. doi: 10.3102/0002831207312909

Zuffianò, A., Alessandri, G., Gerbino, M., Luengo, B. P., Di Giunta, L., Miloni, M., \& Vittorio, G. (2013). Academic achievement: the unique contribution of self-efficacy beliefs in self-regulated learning beyond intelligence, personality traits, and self-esteem. Learning and Individual Differences, 23, 158-162. doi: 10.1016/j. lindif.2012.07.010
Fecha de recepción: septiembre 4, 2015 Fecha de aprobación: marzo 11, 2016 